\title{
Contribuição para a estimativa da freqüência populacional da Persistência Hereditária da Hemoglobina Fetal no Brasil
}

\author{
Estimation of the frequency of Hereditary \\ Persistence of Fetal Hemoglobin in Brazil
}

Vânia Aparecida da Costa 1

Maria Júlia Acedo 1

Newton Carlos Polimeno 1

Carmen Sílvia Bertuzzo 2

\footnotetext{
1 Laboratório Universitário de Análises Clínicas, Hospital Universitário São Francisco,

Universidade São Francisco. Av. São Francisco de Assis 218 Bragança Paulista, SP 12916-900, Brasil. vaniacosta@usf.com.br 2 Departamento de Genética Médica, Faculdade de Ciências Médicas, Universidade Estadual de Campinas. Rua Tessalia Vieira de Camargo 126, Campinas, SP 13081-970, Brasil. bertuzzo@unicamp.br
}

\begin{abstract}
Hereditary Persistence of Fetal Hemoglobin (HPFH) is a benign clinical condition characterized by the synthesis of HbF and which continues without hematological alterations during adult life. Since the function of HPFH in many hemoglobinopathies is that of a severity modulator, it is important to learn its frequency. To obtain this information, a study was conducted on 1,846 blood donors from Bragança Paulista, São Paulo State. Hemoglobin was qualitatively analyzed by hemolytic electrophoresis on agarose gel. Qualitative analysis of $\gamma^{G}$ and $\gamma^{A}$ chains was performed by electrophoresis in polyacrylamide-triton-urea. Two individuals were found to have a high fetal index (0.1\%). The percentage of FHb in one individual was $17 \%$ and in the other 18\%. The gamma G chain was missing in both electrophoretic chains. Cases screened according to laboratory characteristics were of the pancellular hereditary persistence type due to mutation. The frequency in this sample was thus 1/1,000 individuals.
\end{abstract}

Key words Fetal Hemoglobin; Hemoglobinopathies; Abnormal Hemoglobines; Genetics

Resumo Persistência Hereditária de Hemoglobina Fetal (PHHF) é uma condição clinicamente benigna, caracterizada pela síntese contínua da HbF na vida adulta, sem alterações hematológicas. A PHHF funciona como um modulador de gravidade em várias hemoglobinopatias, razão pela qual torna-se importante conhecer a sua freqüencia em nosso meio. Desse modo, por intermédio da análise de 1.846 doadores voluntários de sangue da região de Bragança Paulista, São Paulo, procuramos contribuir para a estimativa dessa freqüência populacional. Realizou-se uma análise qualitativa das hemoglobinas pela eletroforese em gel de agarose. A análise qualitativa das cadeias $\gamma^{G}$ e $\gamma^{A}$ foi realizada pela eletroforese em gel de poliacrilamida-Triton-Uréia. Foram encontrados dois indivíduos com alto índice de hemoglobina fetal (0,1\%). Em um dos indivíduos encontramos um percentual de HbF de $17 \%$ e no outro de 18\%. Nos dois casos a eletroforese de cadeias mostrou uma ausência da cadeia $\gamma^{G}$. Os casos triados, pelas suas características laboratoriais, correspondem à forma pancelular de PHHF, por mutação de ponto. Tal resultado nos permitiu estimar a incidência da PHHF em 1/1.000 indivíduos na população estudada.

Palavras-chave Hemoglobina Fetal; Hemoglobinopatias; Hemoglobinas Anormais; Genética 


\section{Introdução}

As hemoglobinas humanas compreendem um grupo de moléculas com estrutura e propriedades funcionais similares, cuja principal função é o transporte de oxigênio para os tecidos.

Durante a ontogenia, diferentes genes de globinas são sucessivamente expressos, para que a hemoglobina predominante esteja adaptada às diferentes fases do desenvolvimento. Ao nascimento, por exemplo, poucas cadeias $\beta$ são expressas, e a cadeia $\beta$-símile predominante é a cadeia $\gamma$, que se associa com a cadeia $\alpha$ para formar a hemoglobina fetal $\left(\alpha_{2} \gamma_{2}\right)$. Há uma substituição gradual da hemoglobina fetal pelas hemoglobinas adultas $\left(\mathrm{A}_{2} \mathrm{e} \mathrm{A}\right)$, de tal modo que, após o sexto mês de vida do indivíduo, essa substituição estará completa.

A cadeia humana $\gamma$ difere da $\beta$ em 39 dos 146 resíduos aminoácidos. Ao contrário de outras subunidades de globina humana, a cadeia $\gamma$ tem uma heterogeneidade estrutural. Em recém-nascidos, cerca de $2 / 3$ da cadeia $\gamma$ apresentam glicina na posição 136 , enquanto no restante dessas cadeias o aminoácido dessa posição é a alanina. A percentagem da Hemoglobina $\mathrm{F}$ está aumentada na vida adulta em várias anomalias hereditárias, incluindo a talassemia $\beta$, a persistência hereditária de hemoglobina fetal e a anemia falciforme. Em adição, um aumento do nível de hemoglobina fetal pode ser visto em uma variedade de anomalias hematológicas adquiridas, incluindo anemia megaloblástica, anemia aplástica e leucemias, particularmente leucemia mielóide crônica em crianças (Newman et al., 1973). Em homozigotos da talassemia $\beta$ e na anemia falciforme, $o$ aumento percentual da hemoglobina fetal se traduz em um melhor prognóstico da doença.

Persistência Hereditária de Hemoglobina Fetal (PHHF) é uma condição clinicamente benigna, caracterizada pela síntese contínua da HbF na vida adulta, sem alterações hematológicas e com uma relação equilibrada entre a síntese de cadeias $\alpha$ e não- $\alpha$ (Weatheral \& Clegg, 1981; Wood et al., 1979). As PHHF são caracterizadas como condições que possuem um fenótipo similar, mas que resultam de defeitos moleculares múltiplos e complexos.

As PHHF são classificadas pela análise dos parâmetros hematológicos e da lesão molecular existente. Entre os dados hematológicos, são de importância os níveis relativos das cadeias $\gamma^{\mathrm{G}}, \gamma^{\mathrm{A}}, \beta$ e $\delta$, a percentagem de $\mathrm{HbF}$ e seu padrão de distribuição nas hemácias. Com isso, tem-se basicamente classificado as PHHF como pancelulares (aquelas que possuem níveis elevados de síntese de HbF e distribuição uni- forme entre todas as células vermelhas) e heterocelulares (com níveis moderados de hemoglobina fetal e uma distribuição clonal de células com $\mathrm{HbF}$ ).

Em relação à lesão molecular, as PHHF são classificadas em dois grandes grupos: $\mathrm{PHHF}$ por deficiências ou deleções gênicas e $\mathrm{PHHF}$ por mutações de ponto. As deficiências gênicas envolvem grande parte do grupo de genes $\beta \mathrm{e}$ as mutações de ponto são encontradas na região promotora dos genes $\gamma$ (Stamatoyannopoulos et al., 1987). O estudo molecular da PHHF tem tido grande interesse científico por propiciar um entendimento dos mecanismos regulatórios dos genes da hemoglobina. Além disso, como a Persistência Hereditária de Hemoglobina Fetal atua como um modulador de gravidade em várias hemoglobinopatias, torna-se essencial conhecer a sua freqüência em nosso meio. Portanto, nosso objetivo foi contribuir para a estimativa dessa freqüência populacional, com a investigação dessa entidade entre doadores voluntários de sangue da região de Bragança Paulista, São Paulo.

\section{Casuística e métodos}

O estudo foi realizado em 1.846 doadores voluntários de sangue, no período de outubro de 1998 a abril de 1999, no Hemonúcleo da Universidade São Francisco (USF) na Cidade de Bragança Paulista, interior de São Paulo.

A investigação laboratorial foi realizada no Laboratório Universitário de Análises Clínicas do Hospital Universitário São Francisco da USF. As amostras de sangue venoso $(4,5 \mathrm{ml})$ foram colhidas em Vacutainers com EDTA (sal disódico do ácido etilenodinitrotetracético) como anticoagulante, na concentração de $1,5 \mathrm{mg} / \mathrm{ml}$ (Dacie \& Lewis, 1984). Os dados hematológicos foram determinados com a utilização de um equipamento HemoCue Blood Hemoglobin.

Realizou-se uma análise qualitativa das hemoglobinas pela corrida eletroforética do hemolisado em fitas de gel de agarose e tampão barbital 0,05 M pH 8,6, por 20 minutos a $240 \mathrm{~V}$. A hemoglobina fetal foi dosada segundo Betke et al. (1959) e a análise de globinas segundo Alter et al.(1980).

\section{Resultados}

Foram encontrados dois indivíduos não aparentados com alto índice de hemoglobina fetal $(0,1 \%)$. Em um dos indivíduos, encontramos um percentual de $\mathrm{HbF}$ de $17 \%$ (sexo masculino e 
hemoglobina de $16,6 \mathrm{~g} / \mathrm{dl}$ ), e, no outro, de $18 \%$ (sexo feminino e hemoglobina de $13,5 \mathrm{~g} / \mathrm{dl}$ ). Nos dois casos a eletroforese de cadeias mostrou uma ausência da cadeia gama G.

\section{Discussão e conclusão}

Existem poucos trabalhos a respeito da freqüência da hemoglobina fetal na literatura internacional. Tem-se dado prioridade a estudos moleculares, uma vez que a persistência hereditária da hemoglobina fetal é um modulador de gravidade em várias hemoglobinopatias e possibilita um melhor entendimento dos mecanismos regulatórios do grupamento beta. Assim, existem muitos relatos na literatura internacional sobre as mutações que dão origem a essa entidade genética, mas são pouquíssimos os dados sobre sua distribuição populacional.

$\mathrm{Na}$ população brasileira, são conhecidos apenas os dados obtidos entre pacientes do

\section{Agradecimentos}

Os autores agradecem ao Hemonúcleo e ao Laboratório Universitário de Análises Clínicas da Universidade São Francisco pela colaboração prestada na realização desse trabalho.

\section{Referências}

ALTER, B. P.; GOFF, S. C.; EFREMOV, G. D.; GRAVELY, M. E. \& HUISMAN, T. H. J., 1980. Globin chain electrophoresis: A new approach to the determination of the $\gamma \mathrm{G} / \gamma^{\mathrm{A}}$ ratio in fetal haemoglobin and to studies of globin synthesis. British Journal of Haematology, 44:525-534.

BETKE, K.; MARTI, H. R. \& SCHLICHT, I., 1959. Estimation of small percentage of foetal haemoglobin. Nature, 184:1977-1878.

COSTA, F. F.; ZAGO, M. A.; CHENG, G.; NECHTMAN, J. F.; STOMING, T. A. \& HUISMAN, T. H., 1990. The Brazilian type of nondeletional A gamma-fetal hemoglobin has a $\mathrm{C} \rightarrow \mathrm{G}$ substitution at nucleotide - 195 of the a gamma-globin gene. Blood, 76:18961897.

DACIE, J. V. \& LEWIS, S. M., 1984. Pratical Haematology. $6^{\text {th }}$ Ed. Edinburgh/London/Melbourne/New York: Churchill Livinsgstone.

KIMURA, E. M.; DUARTE, D. F; SANTANA, S. C.; BORGES, E.; SILVA, N. M.; GERVASIO, A. S.; COSTA, F. F. \& SONATI, M. F., 2000. Hereditary persistence of fetal hemoglobin (HPFH) an structural alterations of $\gamma$ globin in Brazil. Genetics and Molecular Biology, 23:573.
Hospital das Clínicas da Universidade Estadual de Campinas (UNICAMP), onde encontrou-se a prevalência de 0,1\% (Kimura et al., 2000).

Nossos dados são concordantes com os encontrados em Campinas, mostrando que a freqüência em nossa região seria em torno de $0,1 \%$; por outro lado, em termos de classificação da PHHF, na maior parte dos casos de PHHF de Campinas, havia uma sugestão de que a causa molecular seria deleção gênica, visto que foi detectada a presença dos dois tipos de cadeias $\gamma$. Já nossos casos seriam de mutação de ponto na região promotora do gene $\gamma^{A}$. Tanto em nosso estudo, como no de Kimura (Kimura et al., 2000), não foi detectada a presença da mutação brasileira $\gamma^{\mathrm{A}}$, - $195 \mathrm{C} \rightarrow \mathrm{G}$, descrita por Costa em 1990 (Costa et al., 1990). Esses dados só vêm de encontro à assertiva de que as bases moleculares da Persistência Hereditária da Hemoglobina Fetal são extremamente heterogêneas.

NEWMAN, D. R.; PIERRE, R. V. \& LINMAN, J. W., 1973. Studies on the diagnostic significancy of hemoglobin F level. Mayo Clinic Proceedings, 48:199-202.

STAMATOYANNOPOULOS, G.; NIENHUIS, A. W.; LEDER, P. \& MAJERUS, P. W., 1987. The Molecular Basis of Blood Diseases. Philadelphia: WB Saunders Company.

WEATHERALL, D. J. \& CLEGG, J. B., 1981. The Thalassemia Syndromes. 3th Ed. Oxford: Blackwell.

WOOD, W. G., 1993. Increased HbF in adult life. Baillieres Clinic Hematology, 6:177-213.

WOOD, W. G.; CLEGG, J. B. \&WEATHERALL, D. J., 1979. Hereditary persistence of fetal haemoglobin (HPFH) and delta beta thalassaemia. British Journal of Haematology, 43:509-520.

Recebido em 29 de junho de 2001

Versão final reapresentada em 25 de janeiro de 2002

Aprovado em 3 de abril de 2002 